Research Paper

\title{
Deletion of mitochondrial uncoupling protein 2 exacerbates mitophagy and cell apoptosis after cerebral ischemia and reperfusion injury in mice
}

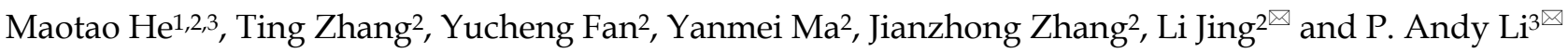 \\ 1. Department of Pathology, General Hospital of Ningxia Medical University, Yinchuan, Ningxia 750004, China. \\ 2. School of Basic Medical Sciences, Department of Pathology, Ningxia Medical University; Ningxia Key Laboratory of Vascular Injury and Repair, Yinchuan, \\ Ningxia 750004, China. \\ 3. Department of Pharmaceutical Sciences, Biomanufacturing Research Institute and Technological Enterprise (BRITE), College of Health and Sciences, North \\ Carolina Central University, Durham, NC 27707, USA. \\ $\square$ Corresponding authors: Dr. P. Andy Li, E-mail: pli@nccu.edu; and Dr. Li Jing, E-mail: 1203220205@qq.com.
}

(C) The author(s). This is an open access article distributed under the terms of the Creative Commons Attribution License (https://creativecommons.org/licenses/by/4.0/). See http://ivyspring.com/terms for full terms and conditions.

Received: 2020.06.23; Accepted: 2020.09.27; Published: 2020.10.16

\begin{abstract}
Objective: Uncoupling protein 2 (UCP2) is a member of inner mitochondrial membrane proteins and deletion of UCP2 exacerbates brain damage after cerebral ischemia/reperfusion (I/R). Nevertheless, its functional role during cerebral $I / R$ is not entirely understood. The objective of present study was to explore the influence of UCP2 deletion on mitochondrial autophagy (mitophagy) and mitochondria-mediated cell death pathway after cerebral I/R

Methods: UCP2- $\%$ and wildtype (WT) mice were subjected to 60 min middle cerebral artery occlusion (MCAO) and allowed reperfusion for 24 hours. Infarct volume and histological outcomes were assessed, reactive oxygen species (ROS) and autophagy markers were measured, and mitochondrial ultrastructure was examined.

Results: Deletion of UCP2 enlarged infarct volume, increased numbers of necrotic and TUNEL positive cells, and significantly increased pro-apoptotic protein levels in UCP2-/- mice compared with WT mice subjected to the same duration of I/R. Further, deletion of UCP2 increased ROS production, elevated LC3, Beclin 1 and PINK1, while it suppressed $\mathrm{p} 62$ compared with respective WT ischemic controls. Electron microscopic study demonstrated the number of autophagosomes was higher in the UCP2-/- group, compared with the WT group.

Conclusions: It is concluded that deletion of UCP2 exacerbates cerebral I/R injury via reinforcing mitophagy and cellular apoptosis in mice.
\end{abstract}

Key words: uncoupling protein 2; cerebral ischemia/reperfusion; mitophagy; autophagy; apoptosis; ROS

\section{Introduction}

Uncoupling protein 2 (UCP2) is a member of inner mitochondrial membrane proteins. It stabilizes the inner mitochondrial membrane potential and reduces the reactive oxygen species (ROS) generation $[1,2]$. Several studies have shown that UCP2 plays important roles in regulating oxidative stress and mitochondrial apoptosis signaling. Abundant evidence also demonstrated that UCP2 plays a significant role in the pathogenesis of cerebral ischemia/reperfusion $(\mathrm{I} / \mathrm{R})$ damage $[3,4,5]$. Our previous studies have shown that deletion of UCP2 gene increases infarct volume after ischemic stroke; and that overexpression of UCP2 reduces ischemic infarct volume [6] and activates cell survival factors [7]. Moreover, deletion of UCP2 has been reported to be associated with mitochondrial damage in cerebral I/R injury [8].

Autophagy is a degradation process of lysosomal metabolism, which is responsible for the degradation of aged or deteriorated organelles and cytoplasmic compositions via lysosomal engulfment into autophagosomes. Mitochondrial autophagy (aka mitophagy) is one of the most important patterns of autophagy. It refers to selective sequestration of mito- 
chondria by autophagosomes, which subsequently were delivered to lysosomes for destruction [9]. The roles of mitophagy in the cerebral I/R have been intensively studied. Recent reports have shown that I/R-induced mitophagy [10]. Nonetheless, the role of mitophagy in cerebral I/ $\mathrm{R}$ is still controversial. While there were studies showed that activation of mitophagy attenuated mitochondrial dysfunction after cerebral I/R [11, 12, 13], others suggested that enhanced mitophagy led to cell death and attenuation of mitophagy protected neurons from cerebral injury $[14,15]$. Therefore, overactivation of mitophagy may degrade essential mitochondria and causes cell death [16]. Recent studies have also shown that mitophagy interacts with ROS and apoptosis in stroke model, especially in PINK1-Parkin-dependent mitochondria $[13,17]$. Our previous studies have revealed that UCP2 attenuates mitochondrial dysfunction and reduces ROS generation from cerebral damage induced by cerebral I/R [6]. However, it is not clear that whether UCP2 could regulate mitophagy in the setting of cerebral I/R injury. Therefore, the objective of this study was to explore the impact of UCP2 deletion on mitophagy and cell death after cerebral I/R.

\section{Methods}

\section{Materials}

2,3,5-Triphenyl Tetrazolium Chloride (TTC) was obtained from Sigma. TUNEL Assay Kit was purchased from Roche (Mannheim, Germany). A Reactive Oxygen Species Assay Kit was obtained from Beyotime (Jiangsu, China). Antibodies against Caspase 3, cleaved Caspase 3, apoptosis proteaseactivating factor-1 (Apaf-1), and cytochrome c (Cyto c) were purchased from Cell Signaling Technology (Danvers, MA). LC3B (ab51520) and p62 (ab109012) antibodies were purchased from Abcam. Beclin1 (11306-1-AP), PINK1 (23274-1-AP), and MnSOD (24127-1-AP) antibodies were purchased from Proteintech. Anti- $\beta$-actin antibody was purchased from Bios (Beijing, China).

\section{Animals and groups}

Male UCP2-/ $(\mathrm{n}=24)$ and wildtype C57BL/ 6 mice $(\mathrm{WT}, \mathrm{n}=48)$ at age of 6-8 weeks were used in this study. All animal procedures were performed in accordance with the NIH Guide for Care and Use of Laboratory Animals and were approved by the Institutional Animal Care and Use Committee at Ningxia Medical University. Breeding pairs of UCP2 $\%$ mice were obtained from the Jackson laboratory and their off-springs were genotyped. The mice were maintained in a specific pathogen-free environment in Laboratory Animal Center of Ningxia
Medical University (Yinchuan, China) with controlled temperature, humidity, and 12:12 hour light and dark cycle. Animals were divided to three groups: Sham group $(\mathrm{n}=24)$, MCAO WT group $(\mathrm{n}=24)$ and MCAO $\mathrm{UCP} 2 \%$ group $(\mathrm{n}=24)$ : $\mathrm{UCP} 2 \%$ mice being subjected to $60 \mathrm{~min}$ MCAO plus $24 \mathrm{~h}$ reperfusion.

\section{Ischemic model}

Cerebral ischemia was induced by occlusion of right middle cerebral artery using a nylon filament. Briefly, animals were anesthetized with isoflurane for induction and maintained at $1.0-1.5 \%$ during the surgical procedures. The internal carotid artery (ICA), external carotid artery (ECA), and the common carotid artery (CCA) were isolated through a ventral cervical midline incision, then the right CCA was ligated. A small incision was made on the CCA and a filament (Doccol corporation, USA), which had a distal cylinder of silicon rubber with a diameter of $0.21 \pm 0.02 \mathrm{~mm}$. After $60 \mathrm{~min}$ occlusion, the filament was withdrawn to achieve recirculation. During the period of surgery, the body temperature of the mice was maintained at approximately $37 \pm 0.5^{\circ} \mathrm{C}$ with a heating pad and lamp and was monitored by a rectal temperature probe. The mice were subjected to a neurological examination after $24 \mathrm{~h}$ reperfusion using Zea-Longa score, where 0 , no neurological deficits; 1 , failure to fully extend left forepaw; 2 , circling to the left; 3, falling to the left; 4, unable to walk spontaneously and exhibiting depressed levels of consciousness.

\section{Measurement of infarct volume and edema}

The mice were sacrificed at $24 \mathrm{~h}$ after MCAO and whole brains were dissected coronally into 1-mm brain slices using a stainless brain matrix (68707, RWD, Shenzhen). The brain slices were immediately incubated with 2\% 2, 3, 5-triphenyltetrazolium chloride (TTC) at $37^{\circ} \mathrm{C}$ for $15 \mathrm{~min}$ and then fixed in $4 \%$ paraformaldehyde. The pale color areas were defined as infarct area. Areas of infarct tissue were measured using NIH Image J software (rsb.info.nih. gov/nih-image) and infarct volumes were calculated from all sections with corrections of intersectional distance. The infarct volume was expressed as percentage of infarct tissue relative to total brain tissue. Brain edema was assessed after $24 \mathrm{~h}$ reperfusion. The relative edema volume (\%) was calculated as: (ipsilateral hemisphere volume contralateral hemisphere volume)/contralateral hemisphere volume $\times 100 \%$.

\section{ROS detection}

The mice brain sections were cultured and incubated with $10 \mu \mathrm{M} / \mathrm{L}$ of dihydroethidium (DHE) for $30 \mathrm{~min}$ at $37^{\circ} \mathrm{C}$. Red precipitations that reflect 
intracellular superoxide production were captured and measured with an Olympus FluoView1000 Laser Scanning Confocal Microscope (using ex/em $\lambda=480$ $\mathrm{nm} / 535 \mathrm{~nm}$ for DHE) and presented as relative fluorescent intensity.

\section{TUNEL staining}

Terminal deoxynucleotidyl transferase mediated dUTP nick-end labeling (TUNEL) staining was used to detect apoptosis (Roche, \#11684795910) according to the manufacturer's protocol. The number of TUNEL-positive cells was counted in five microscopic fields at 400X.

\section{Immunofluorescence staining}

The brain sections were submerged in citrate buffer for antigen retrieval. The protein levels of LC3B (1:100, ab51520, Abcam), Beclin1 (1:200, 11306-1-AP, Proteintech), p62 (1:200, ab109012, Abcam) and PINK1 (1:100, 23274-1-AP, Proteintech) were examined in each group after primary antibody incubation overnight at $4{ }^{\circ} \mathrm{C}$ and secondary antibody incubation at $37^{\circ} \mathrm{C}$ for $45 \mathrm{~min}$. DAPI was used to dye the nuclei. The sections were then viewed under a fluorescence microscope (Olympus). Images were captured at a magnification of $400 \mathrm{X}$.

\section{Western blot}

The ipsilateral brain tissues were homogenized on ice with lysis buffer and protein concentration were measured using BCA method. Equal amounts $(40 \mu \mathrm{g})$ of protein extracts were subjected to $10 \%-12 \%$ SDS-PAGE and transferred to polyvinylidene fluoride membranes (Millipore). The membranes were incubated overnight at $4{ }^{\circ} \mathrm{C}$ with the following primary antibodies individually: anti-MnSOD (1:1000), anti-Apaf-1 (1:1000), anti-Caspase 3 (1:1000), anti-cleaved Caspase 3 (1:1000), anti-Cyto c (1:1000), anti-LC3B (1:1000), anti-Beclin1 (1:1500), anti-p62 (1:2000), and anti-PINK1 (1:1000). Then, the membranes were incubated with secondary antibodies for $1 \mathrm{~h}$ at room temperature. Target protein bands were captured using the BIO-RAD Imaging System with chemiluminescence detection reagents. Semi-quantitative results were obtained by measuring the optical density of the target bands and expressed as the ratio of each targeted protein to $\beta$-actin.

\section{Electron Microscopic Studies}

The brain sections were post-fixed with $4 \%$ glutaraldehyde in $0.1 \mathrm{~mol} / \mathrm{L}$ cacodylate buffer $(\mathrm{pH}$ 7.4). The sections were then soaked in $1 \%$ osmium tetroxide in $0.1 \mathrm{M}$ cacodylate buffers for $2 \mathrm{~h}$ and stained with $1 \%$ aqueous uranyl acetate overnight. Tissue sections were dehydrated in ascending series of ethanol to $100 \%$ followed by acetone and embedded in epoxy resin. Ultrathin sections were counterstained with lead citrate before examination by transmission electron microscope (H7650).

\section{Statistics}

All data are presented as means \pm SD. Statistical analysis was performed using one-way ANOVA with SPSS 19.00. Student's t-test was used to analyze the difference in infarct volume between the WT and $\mathrm{UCP} 2 \%$ groups. Statistical significance was determined as $p<0.05$.

\section{Results}

\section{UCP2 deletion aggravated cerebral I/R damage}

UCP2 deletion enlarged infarct volume, worsened neurological deficit score and increased brain edema after cerebral I/R injury (Figure 1). The infarct volume of the mouse brain was observed by TTC staining (Figure 1A). Focal ischemia of $60 \mathrm{~min}$ duration induced brain infarct in the striatum and the overlaying cortex in WT mice at 24-h of reperfusion. The infarct volume was significantly enlarged in UCP2 $/$ - mice compared with the WT mice (Figure 1B). This result shows UCP2 deletion increased infarct volume. Similarly, UCP2-- mice showed a significant increase in neurological deficit score compared with WT mice (Figure 1C), indicating that neurological deficit scores were correlated to the infarct size. Measurement of brain edema also suggested that $\mathrm{UCP} 2 \%$ significantly increased brain edema compared to WT mice ( $p<0.05$, Figure 1D). Combined, our data demonstrate that UCP2 deletion aggravates ischemic brain damage.

\section{UCP2 deletion exacerbated neural cell injury in I/R brain}

The pathological outcomes assessed by HE and Nissl staining in the cortex after $60 \mathrm{~min}$ of MCAO and $24 \mathrm{~h}$ reperfusion are given in Figure 2. As shown in the Figure 2A upper panel, a few scattered dead neurons were observed in the sham-operated animals. Transient cerebral ischemia resulted in a mildly increased number of dead neurons in the cortex after $24 \mathrm{~h}$ of reperfusion in WT mice $(p<0.01)$. As expected, deletion of UCPs further increased the percentage of dead neurons in the cortex $24 \mathrm{~h}$ after reperfusion in comparison with the WT counterpart (Figure 2A upper panel and 2B). Similarly, Nissl staining showed I/R in WT mice reduced the density of Nissl body and deletion of UCP2 further decreased Nissl body's density compared with ischemic WT mice (Figure 2A lower panel and 2C). These data suggest that the UCP2 deletion induces neural cell damage in $I / R$ brain. 


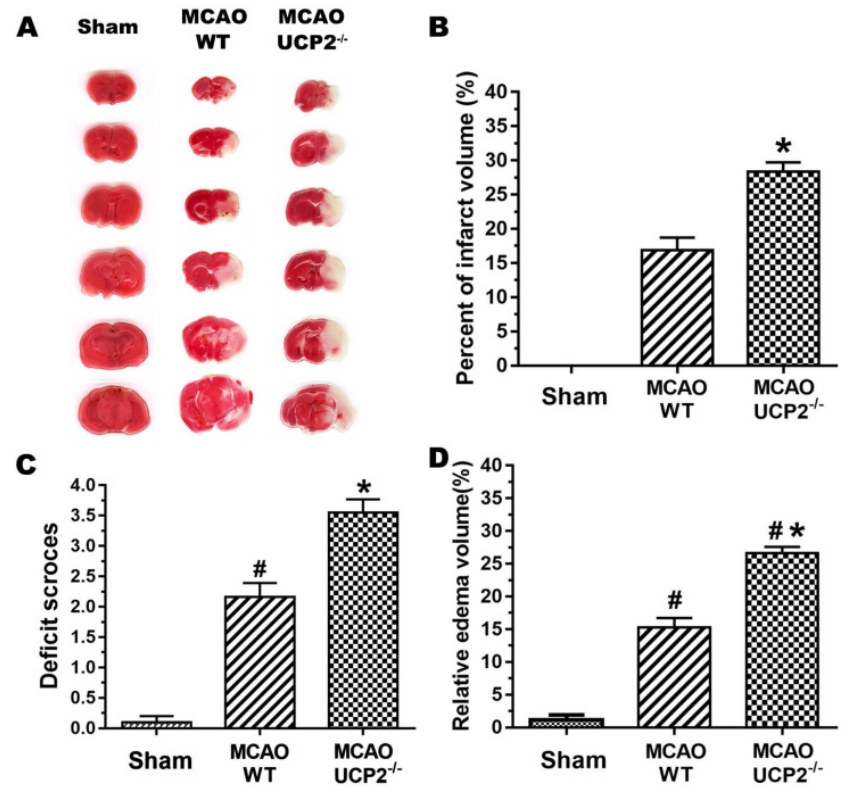

Figure 1. UCP2 deletion aggravated ischemic brain damage. (A) Representative TTC stained brain sections showing infarct volume (white color) at 24-h of reperfusion following $60 \mathrm{~min}$ of MCAO in WT and UCP2 - mice $(\mathrm{n}=5$ in each group). (B) Bar graph summarizes the mean values of cerebral infarction in WT and UCP2 ${ }^{-/}$mice ( $n=5$ in each group). Infarct volume enlarged significantly in UCP2---mice. (C) Assessments of neurological deficits ( $n=24$ in each group). (D) Quantitative analysis of edema volume ( $n=5$ in each group). ${ }^{*} p<0.05$ vs. Sham and $* p<0.05$ vs. WT, respectively.

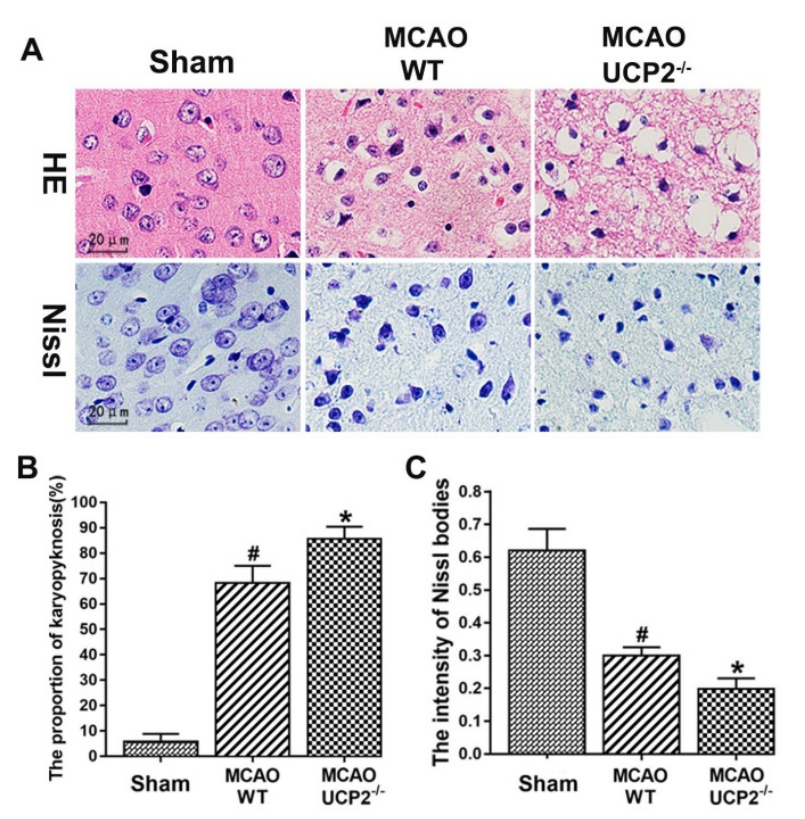

Figure 2. UCP2 deletion enhanced neural cell injury after $\mathbf{I} / \mathbf{R}$ in the brain. (A) Representative images of HE staining (upper panel) and Nissl staining (lower penal). (B) Quantitative summary of pyknotic cells for HE staining ( $n=6$ in each group). (C) The average optical density of Nissl bodies in each group ( $n=6$ in each group). $\#_{p}<0.05$ vs. Sham and $* p<0.05$ vs. WT, respectively.

\section{UCP2 deletion aggravated apoptotic death after I/R injury}

UCP2 deletion increased the number of TUNEL positive cells and activated mitochondria-mediated cell death pathway including Apaf1, Cyto c, and cleaved Caspase 3 after I/R (Figure 3). Cellular apoptosis in ischemic mouse brain was detected with TUNEL staining (Figure 3A). TUNEL staining results revealed that I/R increased the number of TUNEL positive cells in WT mice. UCP2 deletion further elevated the numbers of TUNEL positive cells after $\mathrm{I} / \mathrm{R}$ in UCP2/- compared with the WT counterparts (Figure 3A and 3B). To further examine the effect of $\mathrm{UCP} 2$ deletion on apoptotic pathway after I/R injury in vivo, we measured protein levels of Apaf1, Cyto c, cleaved Caspase 3, and total Caspase 3 in ipsilateral brain tissue collected at $24 \mathrm{~h}$ of reperfusion using Western blotting. The results showed that I/R in WT mice caused significant increases of Apaf1, Cyto c, and cleaved Caspase 3, while total Caspase 3 was not altered. Deletion of UCP2 further elevated the levels of Apaf1, Cyto c, and cleaved Caspase 3 (Figure $3 \mathrm{C}-3 \mathrm{G})$. These results suggest that deletion of UCP2 promotes brain cell apoptosis in cerebral I/R damage.

\section{UCP2 deletion enhanced ROS production after I/R injury}

Because UCP2 has been implicated in dissipating the mitochondrial proton gradient by transporting $\mathrm{H}^{+}$ across the inner membrane, thereby reducing ROS production, we measured the superoxide radical levels using DHE probe in WT and UCP2 $\%$ - mice to assess whether deletion of UCP2 would increase the ROS production. As shown in Figure 4A \& 4B, MCAO induction caused a significant enhancement of superoxide production. Consistently, UCP2 deletion further increased the ROS compared with WT mice after I/R damage. Next, we detected the protein level of MnSOD in mouse brain by Western blot (Figure 4C). The quantitative analysis showed that the MnSOD protein level was lower in the WT group compared with the Sham group, while UCP2 deletion further decreased the level of MnSOD, when it was compared with WT group (Figure 4D). These results suggest that UCP2 deletion enhanced ROS production in the brain after I/R injury.

\section{UCP2 deletion activated autophagy signaling after I/R injury}

Autophagy is one of a major cell death patterns. To investigate whether deletion of UCP2 would activate autophagy signaling pathway after cerebral $\mathrm{I} / \mathrm{R}$ injury, we measured the levels of major autophagy regulators including LC3-B, Beclin-1, p62, and PINK1 using Western blots and immunohistochemistry in brain samples collected from the periinfarct area after $24 \mathrm{~h}$ of reperfusion following $60 \mathrm{~min}$ of MCAO (Figure 5A). The results showed I/R increased the protein levels of LC3 II, Beclin- 1 and PINK1, while it decreased the level of p62, in WT 
animals ( $p<0.05$ vs. Sham). Compared with WT, I/ R in UCP2 $/-$ mice further increased LC3 II, Beclin-1 and PINK1 and decreased p62 (Figure 5A, 5C-5F). Immunolabeling confirmed the trend changes of LC3,
Beclin-1, PINK1 and p62 (Figure 5B). These results suggest that cerebral $\mathrm{I} / \mathrm{R}$ activates autophagy pathway and deletion of UCP2 augments the I/Rinduced activation.
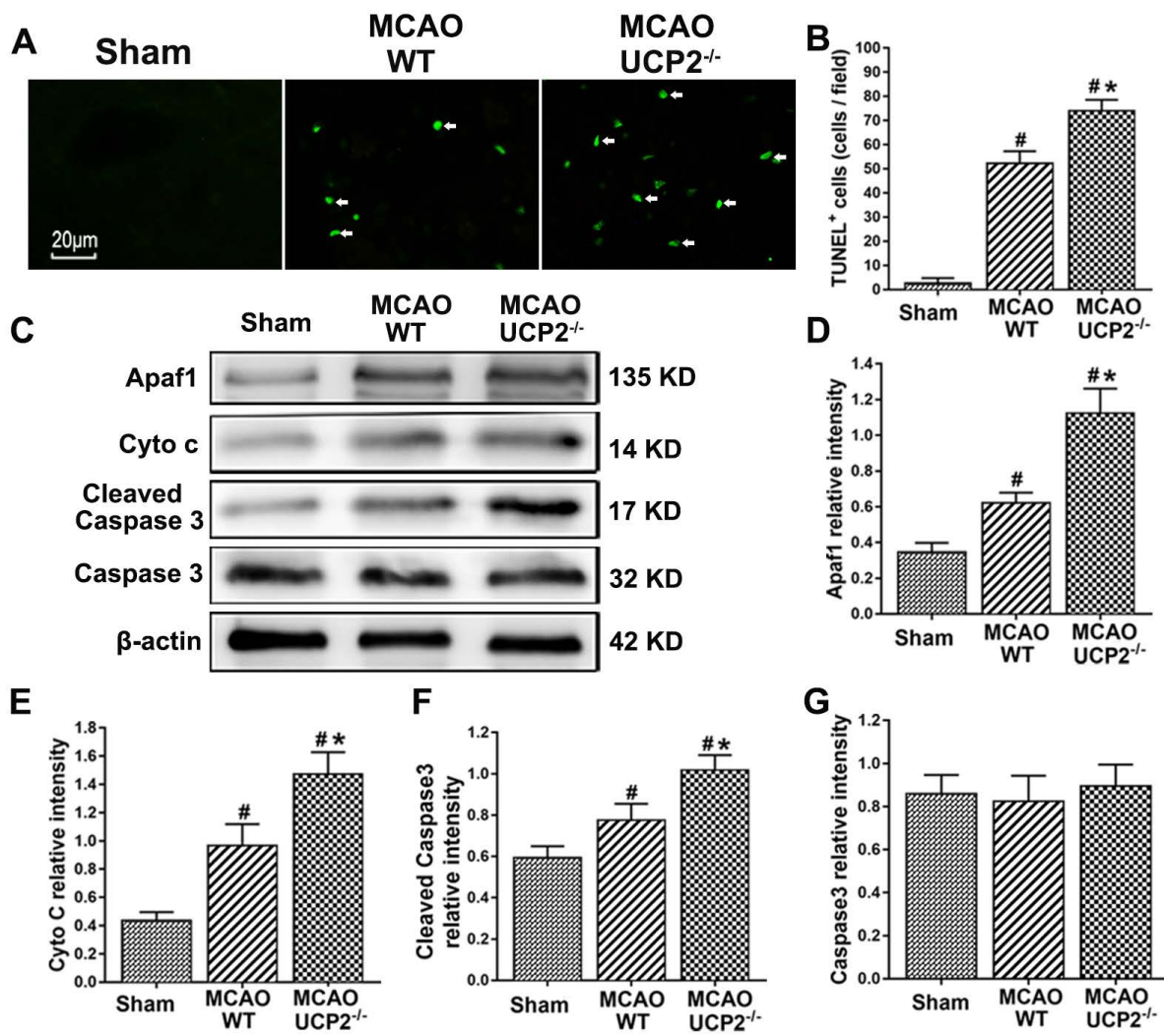

Figure 3. UCP2 deletion aggravated apoptotic death after I/R injury. (A) Representative micrographs showing TUNEL staining for apoptotic cells (green color) among different groups as indicated. Arrows indicate TUNEL-positive cells. Magnification, 400×. Scale bar $=20 \mu \mathrm{m}$. (B) Quantitative determination of TUNEL-positive cells in the brain cortex among different groups as indicated ( $n=6$ in each group). Data are presented as the number of TUNEL-positive cells per high power field (HPF). (C) Representative Western blots of Apaf1, Cyto c, cleaved Caspase 3 and Caspase 3. (D-G) Semi-quantification of Apaf1, Cyto c, cleaved Caspase 3 and total Caspase 3 protein bands, respectively ( $n=6$ in each group). Data are shown as mean \pm SD. ${ }^{*} p<0.05$ vs. Sham, ${ }^{*} p<0.05$ vs. WT, respectively.


Figure 4. UCP2 deletion enhanced ROS production and decreased MnSOD after focal cerebral I/R. (A) Superoxide radicals measured by dihydroethidium (DHE) fluorescent prob in WT and UCP2-- mice ( $n=4$ in each group). Nuclei were labeled with DAPI. Magnification, $400 \times$. Scale bar $=20 \mu m$. (B) Summarized DHE fluorescent intensity ( $n=6$ in each group). Data are presented as means \pm SD. ${ }^{*} p<0.05$ vs. Sham, ${ }^{*} p<0.05$ vs. WT. (C, D) Representative Western blots and quantitative graphs demonstrate the levels of MnSOD in different groups ( $n=6$ in each group). Data are shown as mean \pm SD $(n=6)$. ${ }^{*}<<0.05$ vs. Sham, ${ }^{*} p<0.05$ vs. WT, respectively. 
A

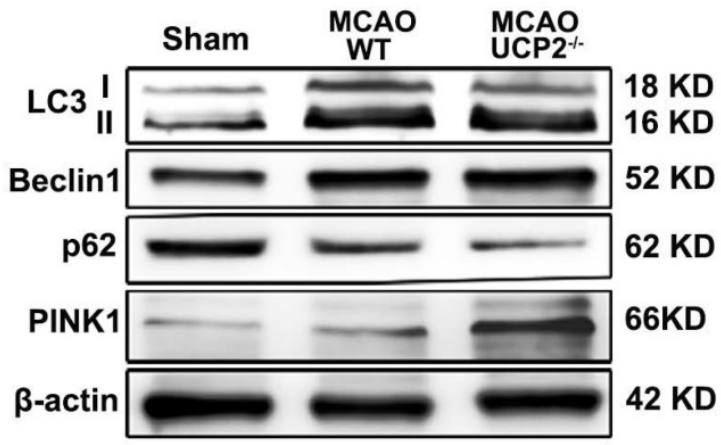

B

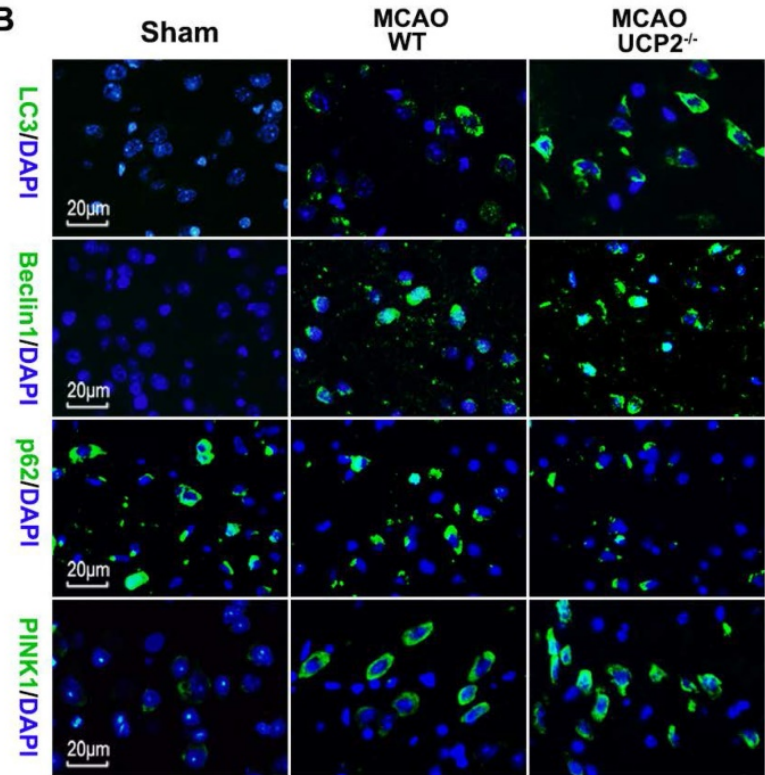

C
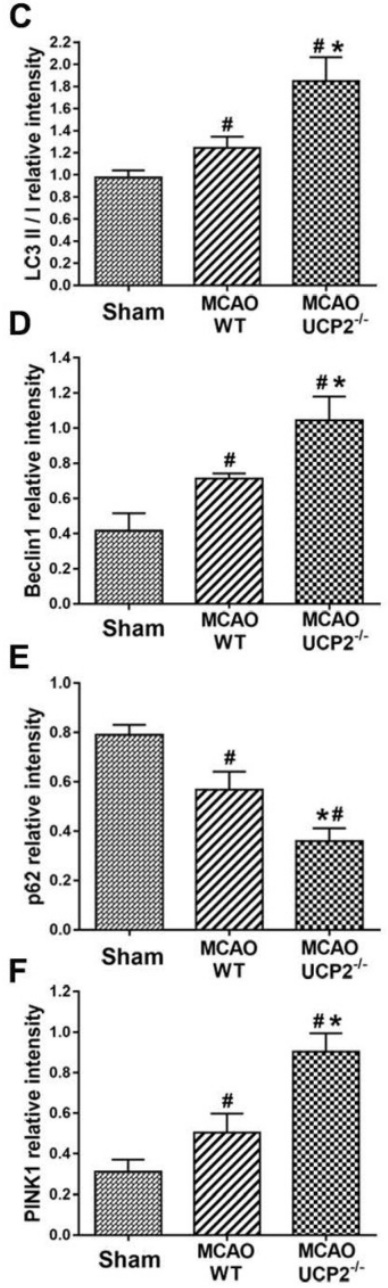

Figure 5. UCP2 deletion activated autophagy pathway after I/R injury. (A) Representative Western blots of LC3-B, Beclin-1, p62, and PINK1 in brain samples collected from peri-infarct area at $24 \mathrm{~h}$ after I/R. (B) Immunohistochemistry of LC3-B, Beclin-1, p62, and PINK1 (green color). Sections were counter labeled with DAPI (Blue color) to mark nuclei. Scale bar $=20 \mu \mathrm{m}$. (C-F) Bar graphs demonstrate semi-quantitations of LC3-II/LC3-I, Beclin-1, p62, and PINK1 ( $\mathrm{n}=6$ in each group). Data are shown as mean \pm SD. ${ }^{\#} p<0.05$ vs. Sham and $* p<0.05$ vs. WT.

\section{UCP2 deletion induced autophagosome accumulation in cortex neurons after $\mathbf{I} / \mathbf{R}$ injury}

It was not clear whether activation of autophagy signaling pathway would actually induce autophagy in the brain after I/R injury. To demarcate the effect of UCP2 deletion on autophagy after cerebral I/R, we performed transmission electron microscopy to examine autophagosomes and autolysosome structures in neurons. As shown in Figure 6, neuron in Sham-operated normal animals showed normal appearance of nucleus $(\mathrm{N})$, abundant endoplasmic reticulum and relatively healthy looking mitochondria $(\mathrm{M})$. In contrast, neuron in the periischemic cortex of the WT ischemic animals displayed mild swollen mitochondria (b) and abnormal autolysosomes (c) at $24 \mathrm{~h}$ after I/ R. Some autophagosomes were also observed in WT group. Furthermore, in the neurons of the UCP2 $/$ - animals, we observed many double layered membrane structures with enclosed mitochondria, which is typically defined as mito- chondrial autophagy or mitophagy. Quantitation of autophagosomes in each animal group revealed that $I / R$ in WT significantly increased the number of autophagosome and UCP2 deletion further increased the number compared with WT ischemic animals (Figure 6B). These results indicate that UCP2 deletion is associated with induction of mitophagy in the brain following I/R injury.

\section{Discussion}

The present study demonstrated that deficiency of UCP2 significantly increased infarct volume and brain edema and caused more severe neurological deficit scores after I/R in UCP2/- mice than WT mice subjected to identical length of I/R. Further, UCP2 deletion enhanced ROS production and cell apoptosis. Moreover, deletion of UCP2 elevated LC3-II/LC3-I ratio, Beclin1 and PINK1 protein contents, suppressed p62 protein level, and increased the number of mitochondria containing autophagosomes compared 
with respective WT ischemic controls. These results suggest that UCP2 deletion may exacerbate cerebral $I / R$ injury via reinforcing mitophagy and cell apoptosis.

UCP2 is a member of inner mitochondrial membrane proteins, which stabilizes the inner mitochondrial membrane potential and reduces ROS generation [1, 2]. UCP2 is widely expressed in various tissues including central nervous system. Emerging evidence suggests that UCP2 plays multiple roles in cerebral I/R injury. On the one hand, it has been found that UCP2 regulates mitochondrial potential, energy balance, and ROS generation; on the other hand, UCP2 has been shown to be associated with cell death and inflammation [18]. It has been reported that overexpressing UCP2 has neuroprotective effects in cerebral stroke [19]. Our data demonstrated that the genetic ablation of UCP2 significantly increased infarct volume, brain edema, and aggravated neurological deficit scores after $I / R$, which is consistent to our previous report and those published in the literature $[6,20,21]$. The previous study has demonstrated that UCP2 induces mitochondrial changes, i.e., increase in mitochondrial density and reduction in mitochondrial size, suggesting that UCP2 expression is associated with a mitochondrial fission process in neurons [22]. And our lab also found that
UCP2 ablation disturbed mitochondrial dynamic balance tilting it towards fission in cerebral I/R damage [23]. Furthermore, our results demonstrated that UCP2 deletion further increased neural cell death in I/ $R$ brain as assessed by histological studies. These results suggest that UCP2 plays an important role in cerebral I/R injury.

Mitochondrial reactive oxygen species (ROS) and apoptosis have been demonstrated to play a critical role in cerebral ischemic/reperfusion injury. ROS are free radicals that can damage DNA, lipids, membranes and organelles, which can lead to activation of cell death processes such as apoptosis [24]. There is abundant evidence showing that the ROS are the by-products of cellular metabolism or of xenobiotic exposure. ROS not only play important roles in cellular signaling but also cause cell damage [25]. It has been reported that UCP2 reduces the ROS formation by preventing mitochondrial membrane hyperpolarization that in turn inhibits mitochondrial electron transport chain. Studies have shown that UCP2 confers protective effects on various stressors by decreasing mitochondrial ROS production in the brain and cardiomyocytes [26-29]. MnSOD is encoded by nuclear genome, synthesized in the cytosol, transported into the mitochondrial matrix post translationally via TOM and TIM23 complexes, and

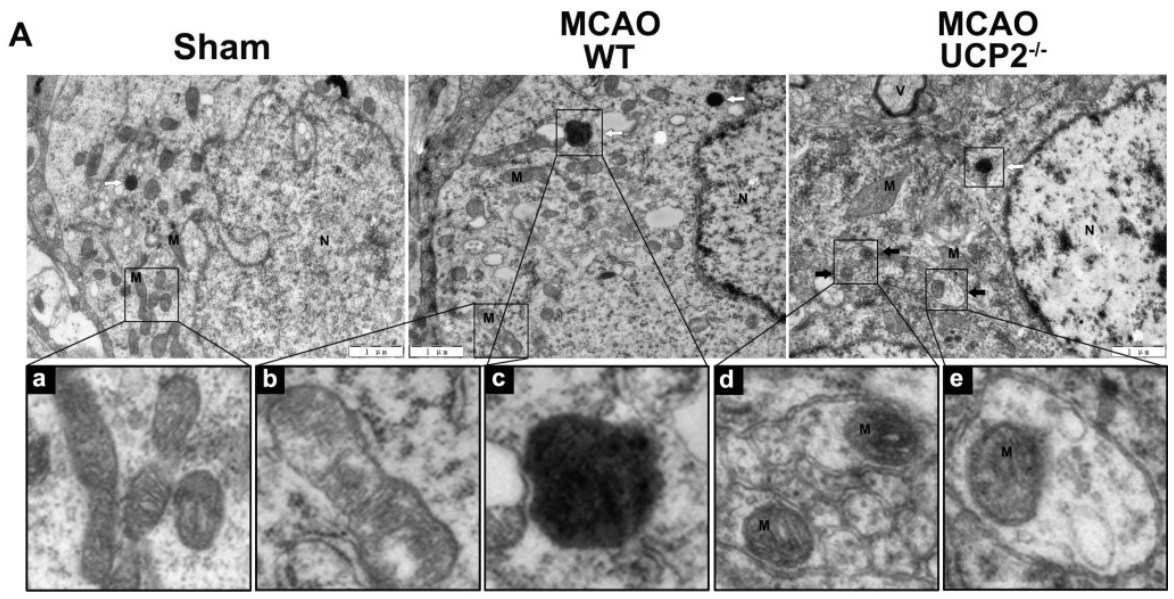

B

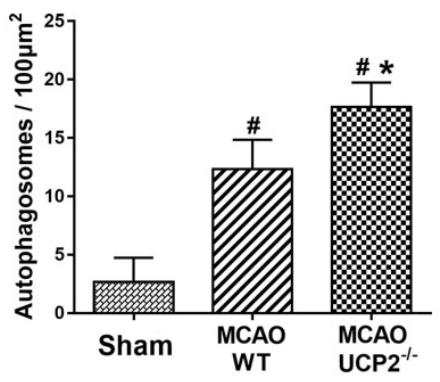

Figure 6. The transmission electron microscope (TEM) examination of ultrastructural changes in cortical neurons 24h after the reperfusion in WT and UCP2 - mice. (A) Representative microphotographs showing autophagosomes in different groups (upper panel). The high magnification images from the cropped rectangle are given in lower panel, in which (a) Normal mitochondrion, (b) mild mitochondrial swollen, (c) autolysosomes, and (d, e) typical mitophagy structure. The characteristics of mitophagy structure (black arrow) and autolysosome structures (white arrow) were observed in neurons $24 \mathrm{~h}$ after MCAO in UCP2 - mice. M, mitochondria; N, nuclei; $\mathrm{V}$, vacuole. scale bar $=1 \mu \mathrm{m}$. (B) Quantification of autophagosomes per $100 \mu \mathrm{m}^{2}$ in neuron $\left(n=3\right.$ in each group). Data are shown as mean \pm SD. ${ }^{*} p<0.05 \mathrm{vs}$. Sham, ${ }^{*} p<0.05 \mathrm{vs}$. WT. 
assembled into active enzyme with the incorporation of a manganese ion in the mitochondrial matrix. The mitochondrial import machinery is a susceptible cellular target of superoxide radicals [30]. The decreased MnSOD enzyme observed in UCP2-/- I/R animals is probably due to increased ROS in UCP2 $\%$ mouse damaged the mitochondrial importing carriers and decreased the transportation of MnSOD from the cytosol into the mitochondrial. Another possibility is that enhance ROS production consumed large amount of MnSOD. In the present study, UCP2 deletion partially induced mitochondrial dysfunction and suppressed MnSOD expression, then enhanced ROS accumulation in the brain after I/R.

Apoptosis is one of the pathways of cell death that are activated by cerebral I/ $R$ injury. There is abundant evidence showing that the mitochondriainitiated apoptotic pathway is activated in response to a variety of cellular stresses, including ROS [31, 32]. Indeed, mitochondria are the major site where most intracellular ROS are produced and accumulation of ROS can trigger the formation of mitochondria permeability transition pore (MPTP). The MPTP allow releases of pro-apoptotic factors such as Apaf-1 and cytochrome c (Cyto c) from the mitochondria to the cytosol, where activating caspases and eventually causing apoptotic cell death $[33,34]$. In this study, we found that cerebral I/ $\mathrm{R}$ increased the protein levels of apoptosis-associated proteins that include Apaf1, Cyto $c$ and cleaved Caspase 3 after I/R injury, and $\mathrm{UCP} 2$ deletion further augmented the elevations of these proteins. These results show that deletion of UCP2 further exacerbates the activation of mitochondria-mediated cell death pathway and the resulting apoptotic cell death in the brain after cerebral ischemic injury. In the meantime, we also found that the presence of high levels of ROS after I/R damage in UCP2/- mice. These results support the hypothesis that UCP2 deletion activates apoptotic cell death probably through increasing ROS after cerebral $I / R$. Our result is supported by previously published data showing that over-expression of UCP2 decreases ROS and apoptosis in cardiomyocytes and in the brain $[7,8,35]$.

Mitochondrial autophagy (mitophagy) is one of the most important types of autophagy in the central nervous system. It refers to selective sequestration of mitochondria by autophagosomes and subsequently delivers them to lysosomes for destruction and material recycle [10]. Previous studies have shown that mitophagy is mainly evident in neurons [36] and astrocytes [37] of the ischemic brain. However, it is controversial whether I/R-triggered mitophagy is beneficial or detrimental to the tissue. On the one hand, inhibition of mitophagy has been shown to exacerbate or reduce ischemic brain injury; on the other hand, enhancement of mitophagy led to cell death [13, 14, 15]. In other words, insufficient or excessive mitophagy may cause cell death due to damaged mitochondrial accumulation or removal of indispensable mitochondria. LC3, Beclin-1, p62, and PINK1 are key regulators for induction of autophagy and mitophagy. LC3 is the most widely used marker for revealing the presence of autophagosomes during autophagy and mitophagy activation. Autophagosome formation involves the conversion of LC3 from LC3-I to LC3-II, suggesting that the LC3-II/ LC3-I ratio closely correlates with the number of autophagosomes [38]. Beclin-1 is another important molecule for mitophagy, which involved in the formation of autophagosomes by membrane recruitment [39]. Additionally, mutations in the mitochondrial Ser/Thr kinase PTEN-induced kinase 1 (PINK1) participates PINK1/Parkin-mediated mitophagy, which is the best-characterized mechanism for elimination of damaged mitochondria by mitophagy in mammalian cells [40, 41]. In damaged mitochondria, PINK1 is stabilized on the outer mitochondrial membrane and recruits Parkin and activates latent E3 ligase activity of Parkin [42, 43]. Parkin activation leads to proteasomal degradation of outer mitochondrial membrane proteins and to mitophagy $[44,45]$. Recent studies have shown that mitophagy interacts with ROS and apoptosis in stroke, especially in PINK1/Parkin-mediated mitophagy [13, 15]. In the present study, we used western blots and immunohistochemistry staining to detect LC3, Beclin-1, and PINK1 expression in the ischemic/reperfusion brain. We found that the LC3-II/LC3-I ratio, Beclin1 and PINK1 protein level were raised after cerebral ischemic/reperfusion. Deletion of UCP2 further increased the levels of LC3-II/LC3-I conversion, Beclin1 and PINK1 compared with WT ischemic control. The p62 (aka SQSTM1), a vital cargo receptor in pink1/parkin mediated mitophagy pathway, is mainly located in the cytosol and forms aggregates [46]. When mitophagy is activated, the p62 protein translocates to the mitochondria and localizes on the sites of autophagosome. It binds with autophagosome localizing protein LC3 and/or ubiquitinated proteins to degrade the autophagy target such as damaged mitochondria [46, 47]. In this study, we detected the expression of p62 by western blot assays. The results showed that the p62 protein level decreased after cerebral I/R and UCP2 deletion further reduced it. The evidence presented in this study indicates that UCP2 deletion reduces accumulation of p62, which is consistent with previous study [11]. The increases of autophagy markers together with mitophagy associated protein marker suggest UCP2 deletion 
increases mitophagy. The follow up electron microscopic study confirmed the existence of mitophagy in UCP2/- animals subjected to I/R.

To study the mitochondrial morphology and to confirm whether activation of mitophagy pathway would lead to increased mitophagy, we performed electron microscopic studies. The results demonstrated that the mitochondrial ultrastructural alterations were much prominent in $\mathrm{UCP} 2 \%$ mice. Further, abnormal mitochondria fused with autophagic vesicles or surrounded by double membranes of typical autophagosomes were observed in UCP2 $\%$ animals. Quantitative analysis of autophagosomes showed that the number of mitochondria-containing autophagosomes in UCP2\%-group was higher than that in the WT group. Considering the increased mitophagy in UCP2 $\%$ mice is accompanied with increased ROS, activated cell death pathway, and augmented neuronal death, we believe that increased mitophagy observed in UCP2 $/-$ animals after I/R is detrimental rather than beneficial. This view is supported by Yang and colleagues who report cerebral ischemic damage is associated with excessive autophagy [48].

In conclusion, the present study demonstrated that deletion of UCP2 gene further exacerbated I/Rinduced brain damage, apoptotic cell death, ROS production and mitophagy. The increased mitophagy is associated with worse ischemic outcome, suggesting excessive mitophagy is harmful in I/R setting.

\section{Highlights}

- Deletion of mitochondrial uncoupling protein 2 (UCP2) exacerbates brain damage after cerebral ischemia/reperfusion injury in mice;

- Deletion of UCP2 aggravates apoptotic death and ROS production after ischemia/reperfusion injury;

- Deletion of UCP2 enhances mitochondrial autophagy (mitophagy) signaling after ischemia/reperfusion injury.

\section{Abbreviations}

UCPs: Uncoupling proteins; UCP2: Uncoupling protein 2; UCP2-/:: UCP2 deletion; I/R: ischemia/ reperfusion; MCAO: middle cerebral artery occlusion; WT: wildtype; ROS: reactive oxygen species; TUNEL: Terminal deoxynucleotidyl transferase mediated dUTP nick-end labeling; TTC: 2,3,5-Triphenyl Tetrazolium Chloride; ICA: internal carotid artery; ECA: external carotid artery; CCA: common carotid artery; DHE: Dihydroethidium.

\section{Acknowledgements}

This study was supported by the Natural Science Foundation of Ningxia Hui Autonomous Region to Maotao He (2019AAC03212), the Ningxia Medical University Research Fund to Maotao $\mathrm{He}$ (XM2016028), Bethune-Merck Diabetes Research Fund to Maotao He and the National Science Foundation of China to Li Jing (No. 81560208). The funders had no role in study design, data collection and analysis, decision to publish, or preparation of the manuscript.

\section{Author contributions}

P. Andy Li and Li Jing conceived and designed the experiments. Maotao He, Ting Zhang, Yucheng Fan and Yanmei Ma performed the experiments. Maotao He and Jianzhong Zhang analyzed the data. Maotao He wrote the original draft. P. Andy Li and Li Jing reviewed and edited the manuscript.

\section{Competing Interests}

The authors have declared that no competing interest exists.

\section{References}

1. Toda C, Diano S. Mitochondrial UCP2 in the central regulation of metabolism. Best Pract Res Clin Endocrinol Metab. 2014; 28(5): 757-764.

2. Lee S, Moon H, Kim G, Cho JH, Lee DH, Ye MB, Park D. Anion Transport or Nucleotide Binding by Ucp2 Is Indispensable for Ucp2-Mediated Efferocytosis. Mol Cell. 2015; 38(7): 657-662.

3. Islam R, Yang L, Sah M, Kannan K, Anamani D, Vijayan C, Fridell YWC. A neuroprotective role of the human uncoupling protein 2 (hUCP2) in a Drosophila Parkinson's disease model. Neurobiol Dis. 2012; 46(1): 137-146.

4. Dutra MRH, Feliciano RDS, Jacinto KR, Gouveia TLF, Brigidio E, Serra AJ, Silva JA. Protective role of UCP2 in oxidative stress and apoptosis during the silent phase of an experimental model of epilepsy induced by pilocarpine. Oxid Med Cell Longev. 2018.

5. Bhattacharya R, Singh P, John JJ, Gujar NL. Oxidative damage mediated iNOS and UCP-2 upregulation in rat brain after sub-acute cyanide exposure: dose and time-dependent effects. Drug Chem Toxicol. 2019; 42(6): 577-584.

6. Haines BA, Mehta SL, Pratt SM, Warden CH, Li PA. Deletion of mitochondrial uncoupling protein-2 increases ischemic brain damage after transient focal ischemia by altering gene expression patterns and enhancing inflammatory cytokines. J Cereb Blood Flow Metab. 2010; 30(11): 1825-1833.

7. Haines B, Li PA. Overexpression of mitochondrial uncoupling protein 2 inhibits inflammatory cytokines and activates cell survival factors after cerebral ischemia. PloS One. 2012; 7(2): e31739.

8. Su J, Liu J, Yan XY, Zhang Y, Zhang JJ, Zhang LC, Sun LK. Cytoprotective effect of the UCP2-SIRT3 signaling pathway by decreasing mitochondrial oxidative stress on cerebral ischemia-reperfusion injury. Int J Mol Sci. 2017; 18(7): 1599.

9. Thomas RL, Gustafsson AB. Mitochondrial autophagy. Circ J. 2013: CJ-13.

10. Tang YC, Tian HX, Yi T, Chen HB. The critical roles of mitophagy in cerebral ischemia. Protein \& cell. 2016; 7(10): 699-713.

11. Li Q, Zhang T, Wang J, Zhang Z, Zhai Y, Yang GY, Sun X. Rapamycin attenuates mitochondrial dysfunction via activation of mitophagy in experimental ischemic stroke. Biochem Biophys Res Commun. 2014; 444 (2): 182-188.

12. Yuan Y, Zheng Y, Zhang X, Chen Y, Wu X, Wu J, Luo J. BNIP3L/NIXmediated mitophagy protects against ischemic brain injury independent of PARK2. Autophagy. 2017; 13(10): 1754-1766

13. Feng J, Chen X, Guan B, Li C, Qiu J, Shen J. Inhibition of peroxynitrite-induced mitophagy activation attenuates cerebral ischemia-reperfusion injury. Mol Neurobiol. 2018; 55(8): 6369-6386.

14. Shi RY, Zhu SH, Li V, Gibson SB, Xu XS, Kong JM. BNIP3 interacting with LC3 triggers excessive mitophagy in delayed neuronal death in stroke. CNS Neurosci Ther. 2014; 20 (12): 1045-1055.

15. Lan R, Zhang Y, Wu T, Ma YZ, Wang BQ, Zheng HZ, Wu JT. Xiao-Xu-Ming decoction reduced mitophagy activation and improved mitochondrial function in cerebral ischemia and reperfusion injury. Behav Neurol. 2018. 
16. Hollville E, Carroll RG, Cullen SP, Martin SJ. Bcl-2 family proteins participate in mitochondrial quality control by regulating Parkin/PINK1-dependent mitophagy. Mol Cell. 2014; 55(3).

17. Lan R, Wu JT, Wu T, Ma YZ, Wang BQ, Zheng HZ, Zhang Y. Mitophagy is activated in brain damage induced by cerebral ischemia and reperfusion via the PINK1/Parkin/p62 signalling pathway. Brain Res Bull. 2018; 142: 63-77.

18. Duffy $\mathrm{CM}, \mathrm{Xu} \mathrm{H}$, Nixon JP, Bernlohr DA, Butterick TA. Identification of a fatty acid binding protein4-UCP2 axis regulating microglial mediated neuroinflammation. Mol Cell Neurosci. 2017; 80: 52-57.

19. Mehta SL, Li PA. Neuroprotective role of mitochondrial uncoupling protein 2 in cerebral stroke. J Cereb Blood Flow Metab. 2009; 29(6): 1069-1078.

20. Mattiasson G, Shamloo M, Gido G, Mathi K, Tomasevic G, Yi S, Nikolich K. Uncoupling protein-2 prevents neuronal death and diminishes brain dysfunction after stroke and brain trauma. Nature Med. 2003. 9(8): 1062-1068.

21. Deierborg T, Wieloch T, Diano S, Warden CH, Horvath TL, Mattiasson G. Overexpression of UCP2 protects thalamic neurons following global ischemia in the mouse. J Cereb Blood Flow Metab. 2008; 28(6): 1186-1195.

22. Toda C, Kim J D, Impellizzeri D, Cuzzocrea S, Liu ZW, Diano S. UCP2 regulates mitochondrial fission and ventromedial nucleus control of glucose responsiveness. Cell. 2016; 164(5): 872-883.

23. He MT, Ma YM, Wang R, Zhang JZ, Jing L, Li PA. Deletion of Mitochondrial Uncoupling Protein 2 Exacerbates Mitochondrial Damage in Mice Subjected to Cerebral Ischemia and Reperfusion Injury under both Normo-and Hyperglycemic Conditions. Int J Biol Sci. 2020; 16(15): 2788-2802.

24. Redza-Dutordoir M, Averill-Bates DA. Activation of apoptosis signalling pathways by reactive oxygen species. Biochim Biophys Acta Mol Cell Res. 2016; 1863(12): 2977-2992.

25. D'Autréaux B, Toledano MB. ROS as signalling molecules: mechanisms that generate specificity in ROS homeostasis. Nat Rev Mol Cell Biol. 2007; 8(10): 813-824.

26. Streck EL, Gonçalves CL, Furlanetto CB, Scaini G, Dal-Pizzol F, Quevedo J. Mitochondria and the central nervous system: searching for a pathophysiological basis of psychiatric disorders. Brazil J Psych.2014; 36(2): 156-167.

27. Hass DT, Barnstable CJ. Uncoupling protein 2 in the glial response to stress: implications for neuroprotection. Neural Regen R. 2016; 11(8): 1197-1200.

28. Zhong H, Liu M, Ji Y, Ma M, Chen K, Liang T, Liu C. Genipin reverses HFDinduced liver damage and inhibits UCP2-mediated pyroptosis in mice. Cell Physiol Biochem. 2018. 49(5): 1885-1897.

29. Wu H, Ye M, Liu D, Yang J, Ding JW, Zhang J, Yang J. UCP2 protect the heart from myocardial ischemia/reperfusion injury via induction of mitochondrial autophagy. J Cell Biochem. 2019. 120(9): 15455-15466.

30. Candas D, Li JJ. MnSOD in oxidative stress response-potential regulation via mitochondrial protein influx. Antioxid Redox Signal. 2014. 20(10): 1599-1617.

31. Hayashi T, Saito A, Okuno S, Ferrand-Drake M, Dodd RL, Chan PH. Damage to the endoplasmic reticulum and activation of apoptotic machinery by oxidative stress in ischemic neurons. J Cereb Blood Flow Metab. 2005. 25(1): 41-53.

32. Deng $S$, Yang $Y$, Han Y, Li X, Wang X, Li X, Wang Y. UCP2 inhibits ROSmediated apoptosis in A549 under hypoxic conditions. PloS one. 2012; 7(1).

33. Garrido C, Galluzzi L, Brunet M, Puig PE, Didelot C, Kroemer G. Mechanisms of cytochrome c release from mitochondria. Cell Death Differ. 2006; 13(9): $1423-1433$

34. Orrenius S. Reactive oxygen species in mitochondria-mediated cell death. Drug Metab Rev. 2007; 39(2-3): 443-455.

35. Yang $\mathrm{K}, \mathrm{Xu}$ X, Nie L, Xiao T, Guan X, He T, Zhao J. Indoxyl sulfate induces oxidative stress and hypertrophy in cardiomyocytes by inhibiting the AMPK/ UCP2 signaling pathway. Toxicol Lett. 2015; 234(2): 110-119.

36. Martinez-Vicente $M$. Neuronal mitophagy in neurodegenerative diseases. Front Mol Neurosci. 2017; 10:64.

37. Quintana DD, Garcia JA, Sarkar SN, Jun S, Engler-Chiurazzi EB, Russell AE, Simpkins JW. Hypoxia-reoxygenation of primary astrocytes results in a redistribution of mitochondrial size and mitophagy. Mitochondrion. 2019; 47: 244-255.

38. Li B, Yao X, Luo Y, Niu L, Lin L, Li Y. Inhibition of autophagy attenuated intestinal injury after intestinal I/R via mTOR signaling. J Surg Res. 2019; 243: 363-370.

39. Geisler S, Jäger L, Golombek S, Nakanishi E, Hans F, Casadei N, Kahle PJ. Ubiquitin-specific protease USP36 knockdown impairs Parkin-dependent mitophagy via downregulation of Beclin-1-associated autophagy-related ATG14L. Exp Cell Res. 2019; 384(2): 111641.

40. Sato S, Furuya N. Induction of PINK1/Parkin-Mediated Mitophagy. Methods in Mol Biol. 2017; I: 9-17.

41. Narendra D, Walker JE, Youle R. Mitochondrial quality control mediated by PINK1 and Parkin: links to parkinsonism. Cold Spring Harb Perspect Biol. 2012; 4(11): a011338.

42. Lazarou M, Jin SM, Kane LA, Youle RJ. Role of PINK1 binding to the TOM complex and alternate intracellular membranes in recruitment and activation of the E3 ligase Parkin. Dev Cell. 2012; 22(2): 320-333.

43. Narendra DP, Jin SM, Tanaka A, Suen DF, Gautier CA, Shen J, Youle RJ. PINK1 is selectively stabilized on impaired mitochondria to activate Parkin. PLoS boil. 2010;. 8(1)

44. Matsuda N, Sato S, Shiba K, Okatsu K, Saisho K, Gautier CA, Kimura M. PINK1 stabilized by mitochondrial depolarization recruits Parkin to damaged mitochondria and activates latent Parkin for mitophagy. J Cell Biol. 2010; 189(2): 211-221.
45. Yoshii SR Kishi C, Ishihara N, Mizushima N Parkin mediates proteasome-dependent protein degradation and rupture of the outer mitochondrial membrane. J Biol Chem. 2011; 286(22): 19630-19640.

46. Liu H, Dai C, Fan Y, Guo B, Ren K, Sun T, Wang W. From autophagy to mitophagy: the roles of P62 in neurodegenerative diseases. J Bioenerg Biomembr. 2017;49(5): 413-422.

47. Johansen T, Lamark T. Selective autophagy mediated by autophagic adapter proteins. Autophagy. 2011; 7(3): 279-296.

48. Yang Y, Gao K, Hu Z, Li W, Davies H, Ling S, Rudd JA, Fang M. Autophagy upregulation and apoptosis downregulation in DAHP and triptolide treated cerebral ischemia. Mediat Inflamm. 2015. 